\title{
Second-order duality for a nondifferentiable minimax fractional programming under generalized $\alpha$-univexity
}

\author{
SK Gupta ${ }^{1 *}$, D Dangar ${ }^{1}$ and Sumit Kumar ${ }^{2}$
}

*Correspondence:

skgiitr@gmail.com

${ }^{1}$ Department of Mathematics,

Indian Institute of Technology,

Patna, 800 013, India

Full list of author information is

available at the end of the article

\begin{abstract}
In this paper, we concentrate our study to derive appropriate duality theorems for two types of second-order dual models of a nondifferentiable minimax fractional programming problem involving second-order $\alpha$-univex functions. Examples to show the existence of $\alpha$-univex functions have also been illustrated. Several known results including many recent works are obtained as special cases.

MSC: 49J35; 90C32; 49N15
\end{abstract}

Keywords: minimax programming; fractional programming; nondifferentiable programming; second-order duality; $\alpha$-univexity

\section{Introduction}

After Schmitendorf [1], who derived necessary and sufficient optimality conditions for static minimax problems, much attention has been paid to optimality conditions and duality theorems for minimax fractional programming problems [2-17]. For the theory, algorithms, and applications of some minimax problems, the reader is referred to [18].

In this paper, we consider the following nondifferentiable minimax fractional programming problem:

$$
\begin{array}{ll}
\text { Minimize } & \psi(x)=\sup _{y \in Y} \frac{f(x, y)+\left(x^{T} B x\right)^{1 / 2}}{h(x, y)-\left(x^{T} D x\right)^{1 / 2}} \\
\text { subject to } \quad g(x) \leq 0
\end{array}
$$

where $Y$ is a compact subset of $R^{l}, f(\cdot, \cdot): R^{n} \times R^{l} \rightarrow R, h(\cdot, \cdot): R^{n} \times R^{l} \rightarrow R$ are twice continuously differentiable on $R^{n} \times R^{l}$ and $g(\cdot): R^{n} \rightarrow R^{m}$ is twice continuously differentiable on $R^{n}, B$, and $D$ are a $n \times n$ positive semidefinite matrix, $f(x, y)+\left(x^{T} B x\right)^{1 / 2} \geq 0$, and $h(x, y)-\left(x^{T} D x\right)^{1 / 2}>0$ for each $(x, y) \in \mathfrak{J} \times Y$, where $\mathfrak{J}=\left\{x \in R^{n}: g(x) \leq 0\right\}$.

Motivated by [7, 14, 15], Yang and Hou [17] formulated a dual model for fractional minimax programming problem and proved duality theorems under generalized convex functions. Ahmad and Husain [5] extended this model to nondifferentiable and obtained duality relations involving $(F, \alpha, \rho, d)$-pseudoconvex functions. Jayswal [11] studied duality theorems for another two duals of (P) under $\alpha$-univex functions. Recently, Ahmad et al. [4] derived the sufficient optimality condition for (P) and established duality relations for

(C) 2012 Gupta et al.; licensee Springer. This is an Open Access article distributed under the terms of the Creative Commons Attribution License (http://creativecommons.org/licenses/by/2.0), which permits unrestricted use, distribution, and reproduction in any medium, provided the original work is properly cited. 
its dual problem under $B$ - $(p, r)$-invexity assumptions. The papers $[2,4-7,11-15,17]$ involved the study of first-order duality for minimax fractional programming problems.

The concept of second-order duality in nonlinear programming problems was first introduced by Mangasarian [19]. One significant practical application of second-order dual over first-order is that it may provide tighter bounds for the value of objective function because there are more parameters involved. Hanson [20] has shown the other advantage of second-order duality by citing an example, that is, if a feasible point of the primal is given and first-order duality conditions do not apply (infeasible), then we may use second-order duality to provide a lower bound for the value of primal problem.

Recently, several researchers $[3,8-10,16]$ considered second-order dual for minimax fractional programming problems. Husain et al. [8] first formulated second-order dual models for a minimax fractional programming problem and established duality relations involving $\eta$-bonvex functions. This work was later on generalized in [10] by introducing an additional vector $r$ to the dual models, and in Sharma and Gulati [16] by proving the results under second-order generalized $\alpha$-type I univex functions. The work cited in [3, 8, $10,16]$ involves differentiable minimax fractional programming problems. Recently, $\mathrm{Hu}$ et al. [9] proved appropriate duality theorems for a second-order dual model of (P) under $\eta$-pseudobonvexity $/ \eta$-quasibonvexity assumptions. In this paper, we formulate two types of second-order dual models for $(\mathrm{P})$ and then derive weak, strong, and strict converse duality theorems under generalized $\alpha$-univexity assumptions. Further, examples have been illustrated to show the existence of second-order $\alpha$-univex functions. Our study extends some of the known results of the literature $[5,6,11,12,14]$.

\section{Notations and preliminaries}

For each $(x, y) \in R^{n} \times R^{l}$ and $M=\{1,2, \ldots, m\}$, we define

$$
\begin{aligned}
J(x)= & \left\{j \in M: g_{j}(x)=0\right\}, \\
Y(x)= & \left\{y \in Y: \frac{f(x, y)+\left(x^{T} B x\right)^{1 / 2}}{h(x, y)-\left(x^{T} D x\right)^{1 / 2}}=\sup _{b \in Y} \frac{f(x, b)+\left(x^{T} B x\right)^{1 / 2}}{h(x, b)-\left(x^{T} D x\right)^{1 / 2}}\right\}, \\
K(x)= & \left\{(s, t, \widetilde{y}) \in N \times R_{+}^{s} \times R^{l s}: 1 \leq s \leq n+1, t=\left(t_{1}, t_{2}, \ldots, t_{s}\right) \in R_{+}^{s},\right. \\
& \left.\sum_{i=1}^{s} t_{i}=1, \widetilde{y}=\left(\widetilde{y}_{1}, \widetilde{y}_{2}, \ldots, \widetilde{y}_{s}\right), \widetilde{y}_{i} \in Y(x), i=1,2, \ldots, s\right\} .
\end{aligned}
$$

Definition 2.1 Let $\zeta: X \rightarrow R\left(X \subseteq R^{n}\right)$ be a twice differentiable function. Then $\zeta$ is said to be second-order $\alpha$-univex at $u \in X$, if there exist $\eta: X \times X \rightarrow R^{n}, b_{0}: X \times X \rightarrow R_{+}$, $\phi_{0}: R \rightarrow R$, and $\alpha: X \times X \rightarrow R_{+} \backslash\{0\}$ such that for all $x \in X$ and $p \in R^{n}$, we have

$$
\begin{aligned}
& b_{0} \phi_{0}\left[\zeta(x)-\zeta(u)+\frac{1}{2} p^{T} \nabla^{2} \zeta(u) p\right] \\
& \quad \geq \alpha(x, u) \eta^{T}(x, u)\left[\nabla \zeta(u)+\nabla^{2} \zeta(u) p\right] .
\end{aligned}
$$

Example 2.1 Let $\zeta: X \rightarrow R$ be defined as $\zeta(x)=e^{x}+\sin ^{2} x+x^{2}$, where $X=(-1, \infty)$. Also, let $\phi_{0}(t)=t+18, b_{0}(x, u)=u+1, \alpha(x, u)=\frac{u^{2}+2}{x+1}$ and $\eta(x, u)=x+u$. The function $\zeta$ is second- 
order $\alpha$-univex at $u=1$, since

$$
\begin{aligned}
& b_{0} \phi_{0}\left[\zeta(x)-\zeta(u)+\frac{1}{2} p^{T} \nabla^{2} \zeta(u) p\right]-\alpha(x, u) \eta^{T}(x, u)\left[\nabla \zeta(u)+\nabla^{2} \zeta(u) p\right] \\
& \quad=2\left(e^{x}+\sin ^{2} x+x^{2}\right)+1.521+3.886(p-1.5)^{2} \\
& \quad \geq 0 \quad \text { for all } x \in X \text { and } p \in R .
\end{aligned}
$$

But every $\alpha$-univex function need not be invex. To show this, consider the following example.

Example 2.2 Let $\Omega: X=(0, \infty) \rightarrow R$ be defined as $\Omega(x)=-x^{2}$. Let $\phi_{0}(t)=-t, b_{0}(x, u)=\frac{1}{u}$, $\alpha(x, u)=2 u$, and $\eta(x, u)=\frac{1}{2 u}$. Then we have

$$
\begin{aligned}
& b_{0} \phi_{0}\left[\Omega(x)-\Omega(u)+\frac{1}{2} p^{T} \nabla^{2} \Omega(u) p\right]-\alpha(x, u) \eta^{T}(x, u)\left[\nabla \Omega(u)+\nabla^{2} \Omega(u) p\right] \\
& =\frac{1}{u}\left[x^{2}+(p+u)^{2}\right] \geq 0 \quad \text { for all } x, u \in X \text { and } p \in R .
\end{aligned}
$$

Hence, the function $\Omega$ is second-order $\alpha$-univex but not invex, since for $x=3, u=2$, and $p=1$, we obtain

$$
\Omega(x)-\Omega(u)+\frac{1}{2} p^{T} \nabla^{2} \Omega(u) p-\eta^{T}(x, u)\left[\nabla \Omega(u)+\nabla^{2} \Omega(u) p\right]=-4.5<0 .
$$

Lemma 2.1 (Generalized Schwartz inequality) Let $B$ be a positive semidefinite matrix of order $n$. Then, for all $x, w \in R^{n}$,

$$
x^{T} B w \leq\left(x^{T} B x\right)^{1 / 2}\left(w^{T} B w\right)^{1 / 2} .
$$

The equality holds if $B x=\lambda B w$ for some $\lambda \geq 0$.

Following Theorem 2.1 ([13], Theorem 3.1) will be required to prove the strong duality theorem.

Theorem 2.1 (Necessary condition) If $x^{*}$ is an optimal solution of problem $(P)$ satisfying $x^{* T} B x^{*}>0, x^{* T} D x^{*}>0$, and $\nabla g_{j}\left(x^{*}\right), j \in J\left(x^{*}\right)$ are linearly independent, then there exist $\left(s^{*}, t^{*}, \tilde{y}\right) \in K\left(x^{*}\right), k_{0} \in R_{+}, w, v \in R^{n}$ and $\mu^{*} \in R_{+}^{m}$ such that

$$
\begin{aligned}
& \sum_{i=1}^{s^{*}} t_{i}^{*}\left\{\nabla f\left(x^{*}, \tilde{y}_{i}\right)+B w-k_{0}\left(\nabla h\left(x^{*}, \tilde{y}_{i}\right)-D v\right)\right\}+\sum_{j=1}^{m} \mu_{j}^{*} \nabla g_{j}\left(x^{*}\right)=0, \\
& f\left(x^{*}, \tilde{y}_{i}\right)+\left(x^{*} T B x^{*}\right)^{1 / 2}-k_{0}\left(h\left(x^{*}, \tilde{y}_{i}\right)-\left(x^{*} T x^{*}\right)^{1 / 2}\right)=0, \quad i=1,2, \ldots, s^{*}, \\
& \sum_{j=1}^{m} \mu_{j}^{*} g_{j}\left(x^{*}\right)=0, \\
& t_{i}^{*} \geq 0\left(i=1,2, \ldots, s^{*}\right), \quad \sum_{i=1}^{s^{*}} t_{i}^{*}=1, \\
& w^{T} B w \leq 1, \quad v^{T} D v \leq 1, \quad\left(x^{* T} B x^{*}\right)^{1 / 2}=x^{* T} B w, \quad\left(x^{* T} D x^{*}\right)^{1 / 2}=x^{* T} D v .
\end{aligned}
$$


In the above theorem, both matrices $B$ and $D$ are positive semidefinite at $x^{\prime \prime}$. If either $x^{*} B x^{*}$ or $x^{*} T x^{*}$ is zero, then the functions involved in the objective of problem (P) are not differentiable. To derive necessary conditions under this situation, for $\left(s^{*}, t^{*}, \widetilde{y}\right) \in K\left(x^{*}\right)$, we define

$$
Z_{\widetilde{y}}\left(x^{*}\right)=\left\{z \in R^{n}: z^{T} \nabla g_{j}\left(x^{*}\right) \leq 0, j \in J\left(x^{*}\right),\right.
$$

with any one of the next conditions (i)-(iii) holds\}.

(i) $\quad x^{* T} B x^{\prime \prime}>0, \quad x^{* T} D x^{\prime \prime}=0$

$$
\begin{aligned}
\Rightarrow \quad z^{T} & \left(\sum_{i=1}^{s^{*}} t_{i}^{*}\left\{\nabla f\left(x^{*}, \tilde{y}_{i}\right)+\frac{B x^{*}}{\left(x^{*} T x^{*}\right)^{1 / 2}}-k_{0} \nabla h\left(x^{*}, \tilde{y}_{i}\right)\right\}\right) \\
& +\left(z^{T}\left(k_{0}^{2} D\right) z\right)^{1 / 2}<0,
\end{aligned}
$$

(ii) $x^{*} B x^{*}=0, \quad x^{*} D x^{*}>0$

$$
\begin{aligned}
\Rightarrow \quad z^{T} & \left(\sum_{i=1}^{s^{*}} t_{i}^{*}\left\{\nabla f\left(x^{*}, \tilde{y}_{i}\right)-k_{0}\left(\nabla h\left(x^{*}, \tilde{y}_{i}\right)-\frac{D x^{*}}{\left(x^{*} T x^{*}\right)^{1 / 2}}\right)\right\}\right) \\
+ & \left(z^{T} B z\right)^{1 / 2}<0,
\end{aligned}
$$

(iii) $\quad x^{*} T x^{*}=0, \quad x^{*} T x^{*}=0$

$$
\Rightarrow \quad z^{T}\left(\sum_{i=1}^{s^{*}} t_{i}^{*}\left\{\nabla f\left(x^{*}, \tilde{y}_{i}\right)-k_{0} \nabla h\left(x^{*}, \tilde{y}_{i}\right)\right\}\right)+\left(z^{T}\left(k_{0}^{2} D\right) z\right)^{1 / 2}+\left(z^{T} B z\right)^{1 / 2}<0 .
$$

If in addition, we insert the condition $Z_{\widetilde{y}}\left(x^{*}\right)=\phi$, then the result of Theorem 2.1 still holds.

For the sake of convenience, let

$$
\psi_{1}(\cdot)=\xi_{1}(\cdot)+\sum_{j=1}^{m} \mu_{j}\left(g_{j}(\cdot)-g_{j}(z)\right)
$$

and

$$
\begin{aligned}
\psi_{2}(\cdot)= & {\left[\sum_{i=1}^{s} t_{i}\left(h\left(z, \tilde{y}_{i}\right)-z^{T} D v\right)\right]\left[\sum_{i=1}^{s} t_{i}\left(f\left(\cdot, \tilde{y}_{i}\right)+(\cdot)^{T} B w\right)+\sum_{j=1}^{m} \mu_{j} g_{j}(\cdot)\right] } \\
& -\left[\sum_{i=1}^{s} t_{i}\left(f\left(z, \tilde{y}_{i}\right)+z^{T} B w\right)+\sum_{j=1}^{m} \mu_{j} g_{j}(z)\right]\left[\sum_{i=1}^{s} t_{i}\left(h\left(\cdot, \tilde{y}_{i}\right)-(\cdot)^{T} D v\right)\right],
\end{aligned}
$$

where

$$
\xi_{1}(\cdot)=\sum_{i=1}^{s} t_{i}\left[\left(h\left(z, \tilde{y}_{i}\right)-z^{T} D v\right)\left(f\left(\cdot, \tilde{y}_{i}\right)+(\cdot)^{T} B w\right)-\left(f\left(z, \tilde{y}_{i}\right)+z^{T} B w\right)\left(h\left(\cdot, \tilde{y}_{i}\right)-(\cdot)^{T} D v\right)\right] .
$$




\section{Model I}

In this section, we consider the following second-order dual problem for (P):

$$
\max _{(s, t, \widetilde{y}) \in K(z)} \sup _{(z, \mu, w, v, p) \in H_{1}(s, t, \widetilde{y})} F(z)
$$

where $F(z)=\sup _{y \in Y} \frac{f(z, y)+\left(z^{T} B z\right)^{1 / 2}}{h(z, y)-\left(z^{T} D z\right)^{1 / 2}}$ and $H_{1}(s, t, \widetilde{y})$ denotes the set of all $(z, \mu, w, v, p) \in R^{n} \times$ $R_{+}^{m} \times R^{n} \times R^{n} \times R^{n}$ satisfying

$$
\begin{aligned}
& \nabla \psi_{1}(z)+\nabla^{2} \psi_{1}(z) p=0, \\
& \sum_{j=1}^{m} \mu_{j} g_{j}(z)-\frac{1}{2} p^{T} \nabla^{2} \psi_{1}(z) p \geq 0, \\
& w^{T} B w \leq 1, \quad v^{T} D v \leq 1, \\
& \left(z^{T} B z\right)^{1 / 2}=z^{T} B w, \quad\left(z^{T} D z\right)^{1 / 2}=z^{T} D v .
\end{aligned}
$$

If the set $H_{1}(s, t, \widetilde{y})=\phi$, we define the supremum of $F(z)$ over $H_{1}(s, t, \widetilde{y})$ equal to $-\infty$.

Remark 3.1 If $p=0$, then using (3.3), the above dual model reduces to the problems studied in $[6,11,12]$. Further, if $B$ and $D$ are zero matrices of order $n$, then (DM1) becomes the dual model considered in [14].

Next, we establish duality relations between primal (P) and dual (DM1).

Theorem 3.1 (Weak duality) Let $x$ and $(z, \mu, w, v, s, t, \tilde{y}, p)$ are feasible solutions of $(P)$ and (DM1), respectively. Assume that

(i) $\psi_{1}(\cdot)$ is second-order $\alpha$-univex at $z$,

(ii) $\phi_{0}(a) \geq 0 \Rightarrow a \geq 0$ and $b_{0}(x, z)>0$.

Then

$$
\sup _{\widetilde{y} \in Y} \frac{f(x, \widetilde{y})+\left(x^{T} B x\right)^{1 / 2}}{h(x, \widetilde{y})-\left(x^{T} D x\right)^{1 / 2}} \geq F(z)
$$

Proof Assume on contrary to the result that

$$
\sup _{\widetilde{y} \in Y} \frac{f(x, \widetilde{y})+\left(x^{T} B x\right)^{1 / 2}}{h(x, \widetilde{y})-\left(x^{T} D x\right)^{1 / 2}}<F(z) .
$$

Since $\tilde{y}_{i} \in Y(z), i=1,2, \ldots, s$, we have

$$
F(z)=\frac{f\left(z, \widetilde{y}_{i}\right)+\left(z^{T} B z\right)^{1 / 2}}{h\left(z, \widetilde{y}_{i}\right)-\left(z^{T} D z\right)^{1 / 2}}
$$

From (3.4) and (3.5), for $i=1,2, \ldots, s$, we get

$$
\frac{f\left(x, \widetilde{y}_{i}\right)+\left(x^{T} B x\right)^{1 / 2}}{h\left(x, \widetilde{y}_{i}\right)-\left(x^{T} D x\right)^{1 / 2}} \leq \sup _{\widetilde{y} \in Y} \frac{f(x, \widetilde{y})+\left(x^{T} B x\right)^{1 / 2}}{h(x, \widetilde{y})-\left(x^{T} D x\right)^{1 / 2}}<\frac{f\left(z, \widetilde{y}_{i}\right)+\left(z^{T} B z\right)^{1 / 2}}{h\left(z, \widetilde{y}_{i}\right)-\left(z^{T} D z\right)^{1 / 2}}
$$


This further from $t_{i} \geq 0, i=1,2, \ldots, s, t \neq 0$ and $\tilde{y}_{i} \in Y(z)$, we obtain

$$
\begin{aligned}
& \sum_{i=1}^{s} t_{i}\left[\left(h\left(z, \tilde{y}_{i}\right)-\left(z^{T} D z\right)^{1 / 2}\right)\left(f\left(x, \tilde{y}_{i}\right)+\left(x^{T} B x\right)^{1 / 2}\right)-\left(f\left(z, \tilde{y}_{i}\right)+\left(z^{T} B z\right)^{1 / 2}\right)\right. \\
& \left.\quad \times\left(h\left(x, \tilde{y}_{i}\right)-\left(x^{T} D x\right)^{1 / 2}\right)\right]<0 .
\end{aligned}
$$

Now,

$$
\begin{aligned}
\xi_{1}(x)= & \sum_{i=1}^{s} t_{i}\left[\left(h\left(z, \tilde{y}_{i}\right)-z^{T} D v\right)\left(f\left(x, \tilde{y}_{i}\right)+x^{T} B w\right)\right. \\
& \left.-\left(f\left(z, \tilde{y}_{i}\right)+z^{T} B w\right)\left(h\left(x, \tilde{y}_{i}\right)-x^{T} D v\right)\right] \\
\leq & \sum_{i=1}^{s} t_{i}\left[\left(h\left(z, \tilde{y}_{i}\right)-\left(z^{T} D z\right)^{1 / 2}\right)\left(f\left(x, \tilde{y}_{i}\right)+\left(x^{T} B x\right)^{1 / 2}\right)\right. \\
& \left.-\left(f\left(z, \tilde{y}_{i}\right)+\left(z^{T} B z\right)^{1 / 2}\right)\left(h\left(x, \tilde{y}_{i}\right)-\left(x^{T} D x\right)^{1 / 2}\right)\right] \quad \text { (using Lemma 2.1 and (3.3)) } \\
< & 0 \quad(\text { from }(3.6)) .
\end{aligned}
$$

Therefore,

$$
\xi_{1}(x)<0=\xi_{1}(z)
$$

By hypothesis (i), we have

$$
b_{0} \phi_{0}\left[\psi_{1}(x)-\psi_{1}(z)+\frac{1}{2} p^{T} \nabla^{2} \psi_{1}(z) p\right] \geq \alpha(x, z) \eta^{T}(x, z)\left\{\nabla \psi_{1}(z)+\nabla^{2} \psi_{1}(z) p\right\}
$$

This follows from (3.1) that

$$
b_{0} \phi_{0}\left[\psi_{1}(x)-\psi_{1}(z)+\frac{1}{2} p^{T} \nabla^{2} \psi_{1}(z) p\right] \geq 0
$$

which using hypothesis (ii) yields

$$
\psi_{1}(x)-\psi_{1}(z)+\frac{1}{2} p^{T} \nabla^{2} \psi_{1}(z) p \geq 0
$$

This further from (2.6), (3.2), and the feasibility of $x$ implies

$$
\xi_{1}(x) \geq-\sum_{j=1}^{m} \mu_{j} g_{j}(x) \geq 0=\xi_{1}(z)
$$

This contradicts (3.7), hence the result.

Theorem 3.2 (Strong duality) Let $x^{*}$ be an optimal solution for $(P)$ and let $\nabla g_{j}\left(x^{*}\right), j \in J\left(x^{*}\right)$ be linearly independent. Then there exist $\left(s^{*}, t^{*}, \tilde{y}^{*}\right) \in K\left(x^{*}\right)$ and $\left(x^{*}, \mu^{*}, w^{*}, v^{*}, p^{*}=0\right) \in$ $H_{1}\left(s^{*}, t^{*}, \tilde{y}^{*}\right)$, such that $\left(x^{*}, \mu^{*}, w^{*}, v^{*}, s^{*}, t^{*}, \tilde{y}^{*}, p^{*}=0\right)$ is feasible solution of (DM1) and the two 
objectives have same values. If, in addition, the assumptions of Theorem 3.1 hold for all feasible solutions $(x, \mu, w, v, s, t, \tilde{y}, p)$ of (DM1), then $\left(x^{*}, \mu^{*}, w^{*}, v^{*}, s^{*}, t^{*}, \tilde{y}^{\prime \prime}, p^{*}=0\right)$ is an optimal solution of (DM1).

Proof Since $x^{*}$ is an optimal solution of $(\mathrm{P})$ and $\nabla g_{j}\left(x^{*}\right), j \in J\left(x^{*}\right)$ are linearly independent, then by Theorem 2.1, there exist $\left(s^{*}, t^{*}, \widetilde{y}^{\prime \prime}\right) \in K\left(x^{*}\right)$ and $\left(x^{\prime \prime}, \mu^{\prime \prime}, w^{\prime \prime}, v^{*}, p^{*}=0\right) \in H_{1}\left(s^{\prime \prime}, t^{*}, \widetilde{y}^{\prime \prime}\right)$ such that $\left(x^{*}, \mu^{*}, w^{*}, v^{*}, s^{*}, t^{*}, \widetilde{y}^{*}, p^{*}=0\right)$ is feasible solution of (DM1) and the two objectives have same values. Optimality of $\left(x^{*}, \mu^{*}, w^{*}, v^{*}, s^{*}, t^{*}, \tilde{y}^{*}, p^{*}=0\right)$ for (DM1), thus follows from Theorem 3.1.

Theorem 3.3 (Strict converse duality) Let $x^{*}$ be an optimal solution to $(P)$ and $\left(z^{*}, \mu^{*}, w^{*}, v^{*}\right.$, $\left.s^{*}, t^{*}, \widetilde{y}^{\prime}, p^{*}\right)$ be an optimal solution to (DM1). Assume that

(i) $\psi_{1}(\cdot)$ is strictly second-order $\alpha$-univex at $z^{*}$,

(ii) $\left\{\nabla g_{j}\left(x^{*}\right), j \in J\left(x^{*}\right)\right\}$, are linearly independent,

(iii) $\phi_{0}(a)>0 \Rightarrow a>0$ and $b_{0}\left(x^{*}, z^{*}\right)>0$.

Then $z^{*}=x^{*}$.

Proof By the strict $\alpha$-univexity of $\psi_{1}(\cdot)$ at $z^{*}$, we get

$$
\begin{gathered}
b_{0}\left(x^{*}, z^{*}\right) \phi_{0}\left[\psi_{1}\left(x^{*}\right)-\psi_{1}\left(z^{*}\right)+\frac{1}{2} p^{*} \nabla^{2} \psi_{1}\left(z^{*}\right) p^{*}\right] \\
>\alpha\left(x^{*}, z^{*}\right) \eta^{T}\left(x^{*}, z^{*}\right)\left\{\nabla \psi_{1}\left(z^{*}\right)+\nabla^{2} \psi_{1}\left(z^{*}\right) p^{*}\right\}
\end{gathered}
$$

which in view of (3.1) and hypothesis (iii) give

$$
\psi_{1}\left(x^{*}\right)-\psi_{1}\left(z^{*}\right)+\frac{1}{2} p^{*} T \nabla^{2} \psi_{1}\left(z^{*}\right) p^{*}>0 .
$$

Using (2.6), (3.2), and feasibility of $x^{*}$ in above, we obtain

$$
\xi_{1}\left(x^{*}\right)>0=\xi_{1}\left(z^{*}\right) .
$$

Now, we shall assume that $z^{*} \neq x^{*}$ and reach a contradiction. Since $x^{*}$ and $\left(z^{*}, \mu^{*}, w^{*}, v^{*}, s^{*}, t^{*}\right.$, $\left.\widetilde{y}, p^{*}\right)$ are optimal solutions to (P) and (DM1), respectively, and $\left\{\nabla g_{j}\left(x^{*}\right), j \in J\left(x^{*}\right)\right\}$, are linearly independent, by Theorem 3.2, we get

$$
\sup _{\widetilde{y}^{*} \in Y} \frac{f\left(x^{*}, \widetilde{y}^{\prime \prime}\right)+\left(x^{*} T B x^{*}\right)^{1 / 2}}{h\left(x^{*}, \widetilde{y}^{\prime \prime}\right)-\left(x^{*} T x^{*}\right)^{1 / 2}}=F\left(z^{*}\right) .
$$

Since $\widetilde{y}_{i} \in Y\left(z^{*}\right), i=1,2, \ldots, s^{\prime \prime}$, we have

$$
F\left(z^{*}\right)=\frac{f\left(z^{*}, \tilde{y}_{i}^{*}\right)+\left(z^{*} T B z^{*}\right)^{1 / 2}}{h\left(z^{*}, \tilde{y}_{i}^{*}\right)-\left(z^{*} T D z^{*}\right)^{1 / 2}} .
$$

By (3.9) and (3.10), we get

$$
\begin{aligned}
& {\left[\left(h\left(z^{*}, \tilde{y}_{i}^{*}\right)-\left(z^{*} T D z^{*}\right)^{1 / 2}\right)\left(f\left(x^{*}, \tilde{y}_{i}^{*}\right)+\left(x^{*} T x^{*}\right)^{1 / 2}\right)\right.} \\
& \left.\quad-\left(f\left(z^{*}, \tilde{y}_{i}^{*}\right)+\left(z^{*} T B z^{*}\right)^{1 / 2}\right)\left(h\left(x^{*}, \tilde{y}_{i}^{*}\right)-\left(x^{*} T D x^{*}\right)^{1 / 2}\right)\right] \leq 0,
\end{aligned}
$$


for all $i=1,2, \ldots, s^{*}$ and $\widetilde{y}_{i}^{*} \in Y$. From $\widetilde{y}_{i}^{*} \in Y\left(z^{*}\right) \subset Y$ and $t^{*} \in R_{+}^{s^{*}}$, with $\sum_{i=1}^{s^{*}} t_{i}^{*}=1$, we obtain

$$
\begin{aligned}
& \sum_{i=1}^{s^{*}} t_{i}^{*}\left[\left(h\left(z^{*}, \widetilde{y}_{i}^{*}\right)-\left(z^{*} T D z^{*}\right)^{1 / 2}\right)\left(f\left(x^{*}, \tilde{y}_{i}^{*}\right)+\left(x^{*} T B x^{*}\right)^{1 / 2}\right)\right. \\
& \left.\quad-\left(f\left(z^{*}, \tilde{y}_{i}^{*}\right)+\left(z^{*} B z^{*}\right)^{1 / 2}\right)\left(h\left(x^{*}, \tilde{y}_{i}^{*}\right)-\left(x^{*} T x^{*}\right)^{1 / 2}\right)\right] \leq 0 .
\end{aligned}
$$

From Lemma 2.1, (3.3), and (3.11), we have

$$
\begin{aligned}
\xi_{1}\left(x^{*}\right)= & \sum_{i=1}^{s^{*}} t_{i}^{*}\left[\left(h\left(z^{*}, \tilde{y}_{i}^{*}\right)-z^{*} T D v^{*}\right)\left(f\left(x^{*}, \tilde{y}_{i}^{*}\right)+x^{*} T B w^{*}\right)\right. \\
& \left.-\left(f\left(z^{*}, \tilde{y}_{i}\right)+z^{*} T B w^{*}\right)\left(h\left(x^{*}, \tilde{y}_{i}^{*}\right)-x^{*} T D v^{*}\right)\right] \\
\leq & \sum_{i=1}^{s^{*}} t_{i}^{*}\left[\left(h\left(z^{*}, \tilde{y}_{i}^{*}\right)-\left(z^{*} T D z^{*}\right)^{1 / 2}\right)\left(f\left(x^{*}, \widetilde{y}_{i}^{*}\right)+\left(x^{*} T B x^{*}\right)^{1 / 2}\right)\right. \\
& \left.-\left(f\left(z^{*}, \tilde{y}_{i}^{*}\right)+\left(z^{*} T B z^{*}\right)^{1 / 2}\right)\left(h\left(x^{*}, \widetilde{y}_{i}^{*}\right)-\left(x^{*} T D x^{*}\right)^{1 / 2}\right)\right] \\
\leq & 0=\xi_{1}\left(z^{*}\right),
\end{aligned}
$$

which contradicts (3.8), hence the result.

\section{Model II}

In this section, we consider another dual problem to $(\mathrm{P})$ :

$$
\max _{(s, t, \tilde{y}) \in K(z)} \sup _{(z, \mu, w, v, p) \in H_{2}(s, t, \tilde{y})} \frac{\sum_{i=1}^{s} t_{i}\left(f\left(z, \tilde{y}_{i}\right)+\left(z^{T} B z\right)^{1 / 2}\right)+\sum_{j=1}^{m} \mu_{j} g_{j}(z)}{\sum_{i=1}^{s} t_{i}\left(h\left(z, \tilde{y}_{i}\right)-\left(z^{T} D z\right)^{1 / 2}\right)},
$$

where $H_{2}(s, t, \widetilde{y})$ denotes the set of all $(z, \mu, w, v, p) \in R^{n} \times R_{+}^{m} \times R^{n} \times R^{n} \times R^{n}$ satisfying

$$
\begin{aligned}
& \nabla \psi_{2}(z)+\nabla^{2} \psi_{2}(z) p=0, \\
& p^{T} \nabla^{2} \psi_{2}(z) p \leq 0, \\
& w^{T} B w \leq 1, \quad v^{T} D v \leq 1, \quad\left(z^{T} B z\right)^{1 / 2}=z^{T} B w, \quad\left(z^{T} D z\right)^{1 / 2}=z^{T} D v .
\end{aligned}
$$

If the set $H_{2}(s, t, \widetilde{y})$ is empty, we define the supremum in (DM2) over $H_{2}(s, t, \widetilde{y})$ equal to $-\infty$.

Remark 4.1 If $p=0$, then using (4.3), the above dual model becomes the dual model considered in $[5,11,12]$. In addition, if $B$ and $D$ are zero matrices of order $n$, then (DM2) reduces to the problem studied in [14].

Now, we obtain the following appropriate duality theorems between (P) and (DM2).

Theorem 4.1 (Weak duality) Let $x$ and $(z, \mu, w, v, s, t, \tilde{y}, p)$ are feasible solutions of $(P)$ and (DM2), respectively. Suppose that the following conditions are satisfied:

(i) $\psi_{2}(\cdot)$ is second-order $\alpha$-univex at $z$,

(ii) $\phi_{0}(a) \geq 0 \Rightarrow a \geq 0$ and $b_{0}(x, z)>0$. 
Then

$$
\sup _{\widetilde{y} \in Y} \frac{f(x, \widetilde{y})+\left(x^{T} B x\right)^{1 / 2}}{h(x, \widetilde{y})-\left(x^{T} D x\right)^{1 / 2}} \geq \frac{\sum_{i=1}^{s} t_{i}\left(f\left(z, \tilde{y}_{i}\right)+\left(z^{T} B z\right)^{1 / 2}\right)+\sum_{j=1}^{m} \mu_{j} g_{j}(z)}{\sum_{i=1}^{s} t_{i}\left(h\left(z, \tilde{y}_{i}\right)-\left(z^{T} D z\right)^{1 / 2}\right)} .
$$

Proof Assume on contrary to the result that

$$
\sup _{\widetilde{y} \in Y} \frac{f(x, \widetilde{y})+\left(x^{T} B x\right)^{1 / 2}}{h(x, \widetilde{y})-\left(x^{T} D x\right)^{1 / 2}}<\frac{\sum_{i=1}^{s} t_{i}\left(f\left(z, \tilde{y}_{i}\right)+\left(z^{T} B z\right)^{1 / 2}\right)+\sum_{j=1}^{m} \mu_{j} g_{j}(z)}{\sum_{i=1}^{s} t_{i}\left(h\left(z, \widetilde{y}_{i}\right)-\left(z^{T} D z\right)^{1 / 2}\right)}
$$

or

$$
\begin{aligned}
& \left(f\left(x, \tilde{y}_{i}\right)+\left(x^{T} B x\right)^{1 / 2}\right)\left[\sum_{i=1}^{s} t_{i}\left(h\left(z, \tilde{y}_{i}\right)-\left(z^{T} D z\right)^{1 / 2}\right)\right] \\
& <\left(h\left(x, \tilde{y}_{i}\right)-\left(x^{T} D x\right)^{1 / 2}\right)\left[\sum_{i=1}^{s} t_{i}\left(f\left(z, \tilde{y}_{i}\right)+\left(z^{T} B z\right)^{1 / 2}\right)+\sum_{j=1}^{m} \mu_{j} g_{j}(z)\right], \\
& \quad \forall \tilde{y}_{i} \in Y(z), i=1,2, \ldots, s .
\end{aligned}
$$

Using $t_{i} \geq 0, i=1,2, \ldots, s$ and (4.3) in above, we have

$$
\begin{aligned}
& \sum_{i=1}^{s} t_{i}\left(f\left(x, \tilde{y}_{i}\right)+\left(x^{T} B x\right)^{1 / 2}\right)\left[\sum_{i=1}^{s} t_{i}\left(h\left(z, \tilde{y}_{i}\right)-z^{T} D v\right)\right] \\
& <\sum_{i=1}^{s} t_{i}\left(h\left(x, \tilde{y}_{i}\right)-\left(x^{T} D x\right)^{1 / 2}\right)\left[\sum_{i=1}^{s} t_{i}\left(f\left(z, \tilde{y}_{i}\right)+z^{T} B w\right)+\sum_{j=1}^{m} \mu_{j} g_{j}(z)\right] .
\end{aligned}
$$

Now,

$$
\begin{aligned}
\psi_{2}(x)= & {\left[\sum_{i=1}^{s} t_{i}\left(f\left(x, \tilde{y}_{i}\right)+x^{T} B w\right)+\sum_{j=1}^{m} \mu_{j} g_{j}(x)\right]\left[\sum_{i=1}^{s} t_{i}\left(h\left(z, \tilde{y}_{i}\right)-z^{T} D v\right)\right] } \\
& -\left[\sum_{i=1}^{s} t_{i}\left(h\left(x, \tilde{y}_{i}\right)-x^{T} D v\right)\right]\left[\sum_{i=1}^{s} t_{i}\left(f\left(z, \tilde{y}_{i}\right)+z^{T} B w\right)+\sum_{j=1}^{m} \mu_{j} g_{j}(z)\right] \\
\leq & {\left[\sum_{i=1}^{s} t_{i}\left(f\left(x, \tilde{y}_{i}\right)+\left(x^{T} B x\right)^{1 / 2}\right)+\sum_{j=1}^{m} \mu_{j} g_{j}(x)\right]\left[\sum_{i=1}^{s} t_{i}\left(h\left(z, \tilde{y}_{i}\right)-z^{T} D v\right)\right] } \\
& -\left[\sum_{i=1}^{s} t_{i}\left(h\left(x, \tilde{y}_{i}\right)-\left(x^{T} D x\right)^{1 / 2}\right)\right]\left[\sum_{i=1}^{s} t_{i}\left(f\left(z, \tilde{y}_{i}\right)+z^{T} B w\right)+\sum_{j=1}^{m} \mu_{j} g_{j}(z)\right] \\
& (\text { from Lemma } 2.1 \text { and }(4.3)) \\
< & \sum_{i=1}^{s} t_{i}\left(h\left(z, \tilde{y}_{i}\right)-z^{T} D v\right) \sum_{j=1}^{m} \mu_{j} g_{j}(x) \quad(\text { using }(4.4)) \\
\leq & 0 \quad\left(\text { since } \sum_{i=1}^{s} t_{i}\left(h\left(z, \tilde{y}_{i}\right)-z^{T} D v\right)>0 \text { and } \sum_{j=1}^{m} \mu_{j} g_{j}(x) \leq 0\right) .
\end{aligned}
$$


Hence,

$$
\psi_{2}(x)<0=\psi_{2}(z)
$$

Now, by the second-order $\alpha$-univexity of $\psi_{2}(\cdot)$ at $z$, we get

$$
b_{0} \phi_{0}\left[\psi_{2}(x)-\psi_{2}(z)+\frac{1}{2} p^{T} \nabla^{2} \psi_{2}(z) p\right] \geq \eta^{T}(x, z) \alpha(x, z)\left\{\nabla \psi_{2}(z)+\nabla^{2} \psi_{2}(z) p\right\}
$$

which using (4.1) and hypothesis (ii) give

$$
\psi_{2}(x)-\psi_{2}(z)+\frac{1}{2} p^{T} \nabla^{2} \psi_{2}(z) p \geq 0
$$

This from (4.2) follows that

$$
\psi_{2}(x) \geq \psi_{2}(z)
$$

which contradicts (4.5). This proves the theorem.

By a similar way, we can prove the following theorems between (P) and (DM2).

Theorem 4.2 (Strong duality) Let $x^{*}$ be an optimal solution for $(P)$ and let $\nabla g_{j}\left(x^{*}\right), j \in$ $J\left(x^{*}\right)$ be linearly independent. Then there exist $\left(s^{*}, t^{*}, \tilde{y}^{*}\right) \in K\left(x^{*}\right)$ and $\left(x^{*}, \mu^{*}, w^{*}, v^{*}, p^{*}=0\right) \in$ $H_{2}\left(s^{*}, t^{*}, \tilde{y}^{*}\right)$, such that $\left(x^{*}, \mu^{*}, w^{*}, v^{*}, s^{*}, t^{*}, \widetilde{y}^{*}, p^{*}=0\right)$ is feasible solution of (DM2) and the two objectives have same values. If, in addition, the assumptions of weak duality hold for all feasible solutions ( $x, \mu, w, v, s, t, \tilde{y}, p)$ of (DM2), then $\left(x^{*}, \mu^{*}, w^{*}, v^{*}, s^{*}, t^{*}, \tilde{y}^{*}, p^{*}=0\right)$ is an optimal solution of (DM2).

Theorem 4.3 (Strict converse duality) Let $x^{*}$ and $\left(z^{*}, \mu^{*}, w^{*}, v^{*}, s^{*}, t^{*}, \widetilde{y}, p^{*}\right)$ are optimal solutions of $(P)$ and (DM2), respectively. Assume that

(i) $\psi_{2}(\cdot)$ is strictly second-order $\alpha$-univex at $z$,

(ii) $\left\{\nabla g_{j}\left(x^{*}\right), j \in J\left(x^{*}\right)\right\}$ are linearly independent,

(iii) $\phi_{0}(a)>0 \Rightarrow a>0$ and $b_{0}\left(x^{*}, z^{*}\right)>0$.

Then $z^{*}=x^{*}$.

\section{Concluding remarks}

In the present work, we have formulated two types of second-order dual models for a nondifferentiable minimax fractional programming problems and proved appropriate duality relations involving second-order $\alpha$-univex functions. Further, examples have been illustrated to show the existence of such type of functions. Now, the question arises whether or not the results can be further extended to a higher-order nondifferentiable minimax fractional programming problem.

The authors declare that they have no competing interests. 


\section{Author details}

${ }^{1}$ Department of Mathematics, Indian Institute of Technology, Patna, 800 013, India. ${ }^{2}$ Indian Institute of Management, Udaipur, 313 001, India.

\section{Acknowledgements}

The authors wish to thank anonymous reviewers for their constructive and valuable suggestions which have considerably improved the presentation of the paper. The second author is also thankful to the Ministry of Human Resource Development, New Delhi (India) for financial support.

Received: 12 April 2012 Accepted: 6 August 2012 Published: 31 August 2012

\section{References}

1. Schmitendorf, WE: Necessary conditions and sufficient conditions for static minmax problems. J. Math. Anal. Appl. 57, 683-693 (1977)

2. Ahmad, I: Optimality conditions and duality in fractional minimax programming involving generalized $\rho$-invexity. Int. J. Manag. Syst. 19, 165-180 (2003)

3. Ahmad, I: On second-order duality for minimax fractional programming problems with generalized convexity. Abstr. Appl. Anal. (2011). doi:10.1155/2011/563924

4. Ahmad, I, Gupta, SK, Kailey, N, Agarwal, RP: Duality in nondifferentiable minimax fractional programming with B-(p,r)-invexity. J. Inequal. Appl. (2011). doi:10.1186/1029-242X-2011-75

5. Ahmad, I, Husain, Z: Duality in nondifferentiable minimax fractional programming with generalized convexity. Appl. Math. Comput. 176, 545-551 (2006)

6. Ahmad, I, Husain, Z: Optimality conditions and duality in nondifferentiable minimax fractional programming with generalized convexity. J. Optim. Theory Appl. 129, 255-275 (2006)

7. Chandra, S, Kumar, V: Duality in fractional minimax programming problem. J. Aust. Math. Soc. A 58, $376-386$ (1995)

8. Husain, Z, Ahmad, I, Sharma, S: Second order duality for minmax fractional programming. Optim. Lett. 3, 277-286 (2009)

9. $\mathrm{Hu}, \mathrm{Q}, \mathrm{Chen}, \mathrm{Y}$, Jian, J: Second-order duality for non-differentiable minimax fractional programming. Int. J. Comput. Math. 89, 11-16 (2012)

10. $\mathrm{Hu}, \mathrm{Q}$, Yang, G, Jian, J: On second order duality for minimax fractional programming. Nonlinear Anal. 12, 3509-3514 (2011)

11. Jayswal, A: Non-differentiable minimax fractional programming with generalized $\alpha$-univexity. J. Comput. Appl. Math. $214,121-135(2008)$

12. Lai, HC, Lee, JC: On duality theorems for a nondifferentiable minimax fractional programming. J. Comput. Appl. Math. $146,115-126(2002)$

13. Lai, HC, Liu, JC, Tanaka, K: Necessary and sufficient conditions for minimax fractional programming. J. Math. Anal. Appl. 230, 311-328 (1999)

14. Liu, JC, Wu, CS: On minimax fractional optimality conditions with invexity. J. Math. Anal. Appl. 219, $21-35$ (1998)

15. Liu, JC, Wu, CS, Sheu, RL: Duality for fractional minimax programming. Optimization 41, 117-133 (1997)

16. Sharma, S, Gulati, TR: Second order duality in minmax fractional programming with generalized univexity. J. Glob. Optim. 52, 161-169 (2012)

17. Yang, XM, Hou, SH: On minimax fractional optimality and duality with generalized convexity. J. Glob. Optim. 31 235-252 (2005)

18. Du, D-Z, Pardalos, PM: Minimax and Applications. Kluwer Academic, Dordrecht (1995)

19. Mangasarian, OL: Second- and higher-order duality in nonlinear programming. J. Math. Anal. Appl. 51, $607-620$ (1975)

20. Hanson, MA: Second order invexity and duality in mathematical programming. Opsearch 30, 311-320 (1993)

doi:10.1186/1029-242X-2012-187

Cite this article as: Gupta et al.: Second-order duality for a nondifferentiable minimax fractional programming under generalized $\alpha$-univexity. Journal of Inequalities and Applications 2012 2012:187.

\section{Submit your manuscript to a SpringerOpen ${ }^{\circ}$ journal and benefit from:}

- Convenient online submission

Rigorous peer review

- Immediate publication on acceptance

- Open access: articles freely available online

- High visibility within the field

- Retaining the copyright to your article 